\title{
The importance of accurate instrumentation for electrical bioimpedance measurements
}

\begin{abstract}
Bioimpedance analysis (BIA) has been successfully used for cancer diagnosis and biomaterial characterizations due to its safety, low cost, effectiveness, portability and applicability. The correct diagnosis depends on the quality of measured data which, in turns, depends on the bioinstrumentation used for capturing and processing the signals. With the advent of innovative materials and integrated circuits, novel approaches in bioinstrumentation have been used to increase both accuracy and precision, and then obtaining a better diagnosis. However, tiny electrodes used to register the signal, such as biosensors, and the increasing number of channels of such a system, impose a great limitation in the electronic, especially at higher frequencies. Further researches in electrical bioimpedance are highly recommended for working with novel and advanced clinical applications in biology and medicine.
\end{abstract}

Volume 7 Issue 3 - 2021

\author{
Pedro Bertemes-Filho \\ Department of Electrical Engineering, State University of Santa \\ Catarina, Brazil
}

\begin{abstract}
Correspondence: Pedro Bertemes-Filho, Rua Paulo Malschitzki 200, Zona Industrial Norte, Joinville, Santa Catarina, Brazil,Tel +55 47 348I7848, Email pedro.bertemes@udesc.br
\end{abstract}

Received: April 30, 2021 | Published: May 12, 2021

Keywords: electrical impedance spectroscopy, bioimpedance, bioinstrumentaion, biomedical sensors, biosignals

\section{Introduction}

The past years have witnessed an unprecedented growth in medical technologies and a new generation of diagnostic tools, characterized by mobility, virtualization, homecare and cost. ${ }^{1}$ The growing demand and need for low cost devices for human tissue characterization with supporting intelligence and technologies (for instance, non-invasive cancer investigations) have arisen unique and evolving opportunities for research in Electrical Impedance Spectroscopy (EIS) technique. EIS is based on measurements of an electrical impedance spectrum tissues and extraction of equivalent electrical parameters for diagnostic purposes ${ }^{2}$ and statistical analysis. It is considered a cheap, robust, non-ionizing and non-invasive technique, but requires some expertise for collecting data from materials under study. Measured data has to be reliable and should thus be collected in a proper manner in order to truly represent the material properties. However, stray capacitance and electronic noises affects the data and, consequently, weaken the use of this technique for medical applications. ${ }^{3}$ Higher input impedance amplifiers, noise feedback control, ${ }^{4}$ wider frequency range current source ${ }^{5}$ and stray capacitance attenuators ${ }^{6,7}$ are some examples of innovative solutions to improve the bioinstrumentation performance. The objective of this short communication is to define and purpose novel research themes using Bioimpedance Analysis (BIA) which contributors might address. It also brings the attention of new trends and innovative instrumentation techniques for resulting into an accurate bioimpedance spectroscopy system working in a wide frequency range.

\section{Accuracy and precision impacts}

The more accurate is the bioinstrumentation involved in the technique the more trustable will be the generated and measured signals and consequently the diagnosis. As an electrical properties extractor tool, this is also very important for obtaining the correct diagnosis from impedance data. Many EIS systems have also combined impedance spectra with other clinical data, ${ }^{8}$ as for example imaging the lung ventilation and perfusion. ${ }^{9}$ Misunderstood diagnosis can easily be obtained if impedance data are not precisely measured and post-processed.
Measuring electrical bioimpedance in a wide frequency range from $10 \mathrm{~Hz}$ to $10 \mathrm{MHz}$ is very difficult in terms of electronics. Most recent commercial electrical components, such as instrumentation amplifiers, buffers and operational amplifiers do not have the ideal characteristics for working in a BIA device, specially at higher frequencies $(>1 \mathrm{MHz})$. In order to overcome the limitations of integrated circuits (IC), prominent solutions have been using current conveyors circuits developed with bipolar/CMOS/Bi-CMOS architectures. ${ }^{10}$ Other recent advances are related to the improvements of the voltage controlled current source for driving the biological loads, such as the modified Howland current source (HCS). ${ }^{11}$ Special HCS circuits with error feedback control can be designed for driving high loads in a wider frequency range. ${ }^{12}$ Some bioimpedance analyzers use multiple electrodes, then it requires the use of multiplexers which, in turns, add more stray capacitance in the measuring system. Multiple current sources, error feedback control and buffer isolation might be of interested for improving the BIA accuracy.

\section{Conclusion}

It was showed that accuracy is an important matter to be addressed if novel and innovative applications of EIS and BIA (bioimpedance analysis) are requested in a future time. Students and research groups might have the opportunity to get more from this technique, then more clinics and hospitals may use it as a commercial tool in theirs daily basis. It was observed that the specific themes for bioimpedance analysis might include current conveyors, Howland source topology, negative impedance converter, multisource drive system, novel analog switch, novel instrumentation amplifier and feedback control circuit. It can be concluded that novel approaches in bioinstrumentation together with best practice are the key factors for working towards an advanced understanding of the knowledge base that we will collectively draw upon in the years ahead to meet future challenges.

\section{Acknowledgments}

I thank the State University of Santa Catarina (UDESC) and the Research Foundation of Santa Catarina (FAPESC) for the institutional and financial support, respectively. 


\section{Conflicts of interest}

I declare no conflict of interest.

\section{References}

1. Bertemes-Filho P, Bayford R, Frerichs I. Topical issues in electrical impedance tomography and bioimpedance application research. Physiological Measurement. 2020(41):120301.

2. Grimnes S, Martinsen O. Bioimpedance and Bioelectricity Basics. 3rd edn. Academic Press; 2014.

3. Pliquett U, Frense D, Schönfeldt M, et al. Testing miniaturized electrodes for impedance measurements within the $\beta$-dispersion - a practical approach. Journal of Electrical Bioimpedance. 2010(1):41-55.

4. Sirtoli VG, Vincence VC, Bertees-Filho P. Mirrored enhanced Howland current source with feedback control. Review of Scientific Instruments. 2019(90):024702.

5. Morcelles K, Sirtoli V, Bertemes-Filho P, et al. Howland current source for high impedance load applications. Review of Scientific Instruments. 2017(88):114705.

6. Dutra D, Bertemes-Filho P. Extracting parasite effects of electrical bioimpedance measurements. Journal of Electrical Bioimpedance. 2018(9):115-122.
7. Dutra D, Bertemes-Filho P. Modeling parasitic effects with a RLCRSC model in electrical impedance measurements. Journal of Physics: Conference Series. 2019(1272):012019.

8. Cavalieri R, Bertemes-Filho P. Plant tissue differentiation using electrical impedance spectroscopy with deep neural networks. International Journal of Biosensors \& Bioelectronics. 2020(6):20-23.

9. Adler A, Amanato MB, Arnold JH, et al. Whither lung EIT: where are we, where do we want to go and what do we need to get there? Physiological measurement. 2012;33(5):679.

10. Bertemes-Filho P, Vincence VC, Santos MS, et al. Low power current sources for bioimpedance measurements: A comparison between Howland and integrated CMOS OTA circuits. Journal of Electrical Bioimpedance. 2012(3):66-73

11. Beretemes-Filho P, Negri LH, Vincence VC. Designing a mirrored Howland circuit with a particle swarm optimisation algorithm. International Journal of Electronics. 2015(1):150812050133003.

12. Morecelles K, Negri LH, Bertemes-Filho P. Design of Howland current sources using differential evolution optimization. Journal of Electrical Bioimpedance. 2020;11: 96-100. 